\title{
Moving Towards Reliability-Centred Management of Energy, Power and Transportation Assets
}

\author{
Kang Seng Seow, Loc K. Nguyen*, Kelvin Tan, Kees-Jan Van Oeveren \\ Clean Technology Centre, DNV GL - Energy, 118227, Singapore
}

\begin{tabular}{l} 
A R T I C L E I N F O \\
\hline Article history: \\
Received: 12 April, 2017 \\
Accepted: 02 May, 2017 \\
Online: 17 May, 2017 \\
\hline Keywords: \\
Physical Asset Management \\
Risk-based Asset Management \\
Reliability Centered Maintenance
\end{tabular}

\begin{abstract}
A B S T R A C T
Infrastructure Asset Management was first adopted in 1980s for challenges faced by the utilities, road and rail transport systems, mining, process and manufacturing industries in asset life cycle value optimization. International standards ISO 55000 and PAS 55 were subsequently developed to provide a basis for a structured methodology in the development of Asset Management System. This methodology with the basic framework is considered inadequate in addressing the changing and challenging environment faced by the Energy, Power and Transportation sectors. This paper introduces the concept of Reliability-Centred Management System that could leverage on the advancement in Information and Communication Technologies and the entry of the 'Big Data' and 'Smarter Grid' era.
\end{abstract}

\section{Introduction}

Incidents in the late 1980's, such as the North Sea Piper Alpha disaster that caused 167 fatalities, and the ensuing oil price crash and the falling levels of service, escalating costs and poor planning in the public sector of Australia and New Zealand has necessitate a radical change in managing the assets, such as the oil platforms and the public infrastructures. The lessons learnt from these widely different environments have been remarkable similar, with significant convergence of conclusions. This has led to the approach of asset life cycle value optimization for the utilities, road and rail transport systems, mining, process and manufacturing industries at an accelerating rate. [1]

On the other hand, international standards, such as PAS 55 and ISO 55000 developed in the last decade provide a basis for a structured methodology in the development of Asset Management System. However, this methodology with the basic framework is not adequate in addressing the changing and challenging environment faced by the power utilities. How utilities manage their assets is undergoing profound changes. Asset Management approaches and analytical tools have finally matured to the point where decisions can be based on data and facts - not just "gut feel" instincts and intuition.

\section{Current Asset Management Concepts}

Facing with the changing and challenging environment brought about in the global economic and local political pressures,

"Corresponding Author: Loc K. Nguyen, Clean Technology Centre, DNV GL Energy, 118227, Singapore | Email: khanh.loc.nguyen@dnvgl.com the Asset Management mind-set for the energy, power and transportation sectors needs to focus on:

- $\quad$ Prudent performance targets, not maximum performance;

- $\quad$ Risk management not risk avoidance; and

- $\quad$ Spending justified by performance improvement and risk mitigation not blanket budgets.

The quality of assets managed by the energy, power and transportation sectors cannot therefore be assured by simple compliance with the general ISO 9001 management system standard [2]. This is because ISO 9001 provides little guidance on what constitutes good Asset Management practice with regard to capital-intensive infrastructures with relatively long asset lives.

The crux of Asset Management in these sectors is to have an integrated, whole-life and risk based approach. The issues faced by the energy, power and transportation sectors are many and varied. These sectors are required by their stakeholders to procure, operate, maintain and subsequently replace their assets at acceptable risk levels with satisfactory quality of service and reasonable return of capital investments. These assets comprise complex assets which typically have operational lives of several decades. At the same time, the sectors have to avoid unnecessary investments planning while ensuring the reliability, security and quality of services provided by these assets.

Cost-effective operation and maintenance of the assets are particularly important in the management of the assets throughout 
the useful life of these assets. Figure 1 shows the possible strategies that can be adopted in the maintenance of the assets.

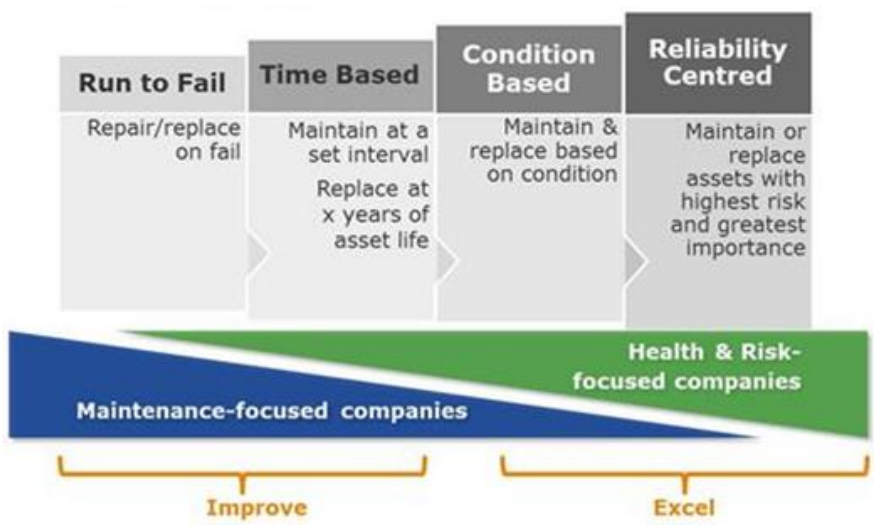

Fig. 1. Maintenance Strategy Evolution

\section{Technical Asset Management System}

Figure 2 shows a typical step-wise implementation approach that has been developed for Asset Management Systems of energy, power and transportation sectors under ISO 55000. [3,4]

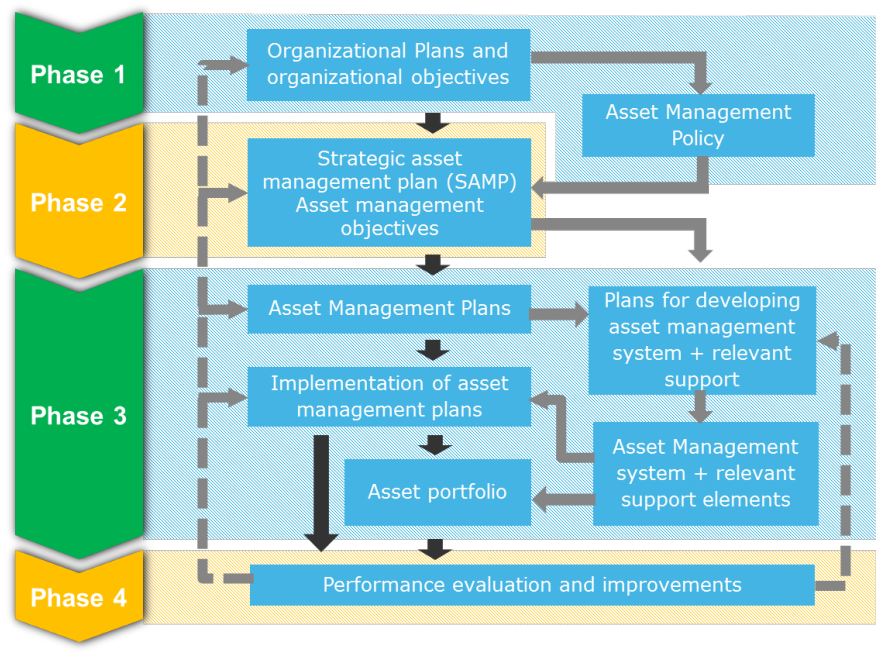

Fig. 2. Typical Four-Phase Approach for Asset Management System Implementation

Phase 1 is to have an in-depth understanding of the organizational objectives and goals for meeting both the internal and external requirements. Demand growth, failure behaviour and other major trends and drivers impacting the power utility with regard to reliability, financial, safety, social, political, legal, and regulatory perspective are the most common external considerations.

Phase 2 is the scoping of the Asset Management System. In this phase, the power utility needs to develop Asset Management objectives and a Strategic Asset Management Plan (SAMP) which are in alignment with organizational objectives. The SAMP is developed in consideration of expectations and needs from all stakeholders, and includes business values and Key Performance Indicators (KPIs); decision making processes and criteria; boundaries of the Asset Management System with scope; and plans and programs to achieve the Asset Management System objectives.
Phase 3 is the resource planning and implementation phase, which is dedicated to the actual implementation of the Asset Management System including the required resources. This involves the development of processes and procedures, development of the right technical and IT tools and systems, and competence building, and awareness \& communication on a regular basis.

The technical scope of Asset Management needs to be aligned with the asset life cycle. In particular, different Asset Management practices should address the following four different stages of life span of the assets: - specification and design, commissioning, maintenance and operation, and retiring.

According to Kristian Steenstrup, VP and Gartner Fellow, Energy and Utilities, "There are many strategies to improve reliability. Rather than thinking of them as conflicting or contradictory, develop a road map that matches the best tool to the characteristics of specific assets."

Technical Asset Management is therefore focusing on complex assets, where maintenance and analytics are used to support the reliable operation of the asset and to extend its useful life.

Technical Asset Management methodologies in each stage of the life span of the asset are shown in the Figure 3. The methodologies have to be selected to manage the asset in each stage properly in order to reduce the risk, achieving the real advantages and benefits of the Asset Management System.

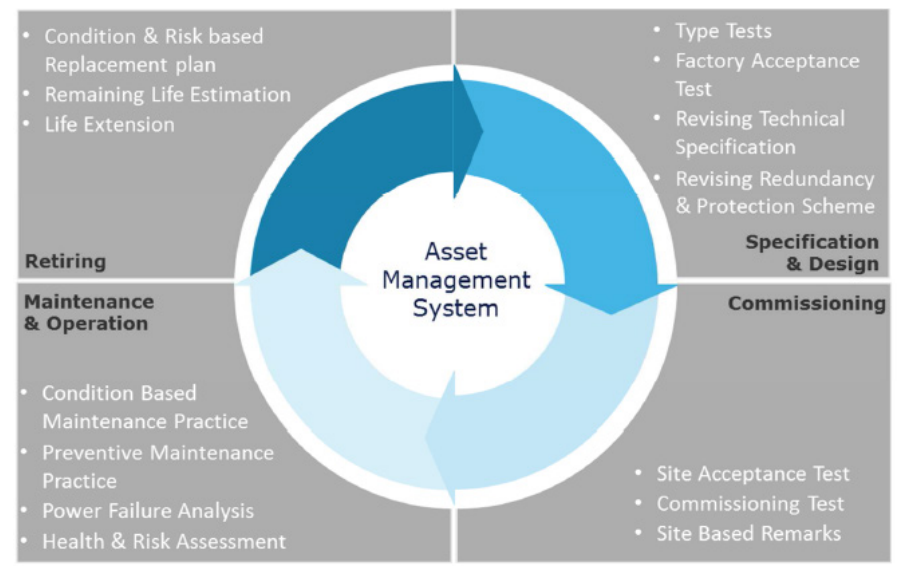

Fig. 3. Technical Asset Management Methodologies in Different Stages of Asset Life

Phase 4 is the evaluation and improvement phase as the Asset Management System should be continuously evaluated for possible improvements and corrections to be in alignment with inherent objectives. In this respect, systematic measurement of performance with quantitative and qualitative indices can contribute to the Asset Management System improvement with SMART targets for a specific process or the system.

However, technical and financial constraints of the energy, power and transportation sectors usually hinder Asset Management System realization as planned. In order to overcome such constraints and to plan the Asset Management System in a tailor-made manner for short/long-term goals, a technical gap analysis can be carried out to understand the gap between current practices with technical competence and required scenario's, prioritizing required technologies, tools or competences in an appropriate order. 
This technical gap analysis not only enables the energy, power and transportation sectors in assessing their current practice, but can also be used to list and prioritize the technologies and methodologies to which the energy, power and transportation sectors have to pay attention to within their objectives and resource boundary in order to plan for the resourcing of required tools on a short or long-term basis. Asset Management supporting Information Technology (IT) tools therefore play an important role to support the technical performance of the Asset Management System in order to maintain, control, and analyse the information and its trend over the time, understanding of assets and their risks throughout their lifecycle. [5]

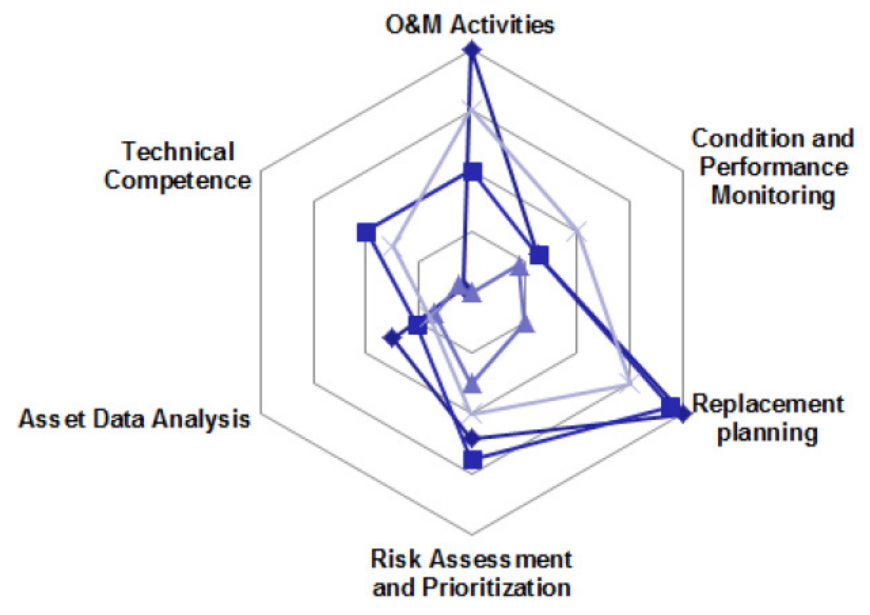

Fig. 4. Example of a Technical Gap analysis

Since sound decisions can be highly dependent on having a high quality of information, support by efficient IT tools for data acquisition and analysis is therefore critical. In this sense, some considerations of IT tools are

1. Scalability: IT tools should accommodate all assets the power utility likes to include for monitoring and maintenance;

2. Lifecycle Coverage: IT tools should cover all available information and its trend over the lifecycle of the asset;

3. Compatibility: When energy, power and transportation sectors decide to adapt new condition assessment or monitoring software solution, IT tools should communicate core condition information on assets to one another; and

4. Flexibility: IT tools should support any specific methods and information as a template in which the energy, power and transportation sectors like to add or remove at any time.

In recent years, more and more energy, power and transportation sectors are considering to utilize on-line monitoring devices to extract meaningful dynamic condition parameters such as Partial Discharge, Tangent Delta, or Dissolved Gas Analysis for their power assets and other parameters such as vibration, temperature, corrosion, etc. for other critical assets.

\section{Asset Management Decision Support Modeling Using Health Indexing}

With the rapid growth of demand and the maturing electricity sectors in Asia, energy, power and transportation sectors are facing many challenges in managing their power assets, such as increasing demands from stakeholders with regard to safety, reliability, environmental impact and financial results, aging assets with increased risks of failure, and, as a result, a decreased level of reliability and (possibly) safety, etc. In planning maintenance and replacement of the vast number of growing assets, asset managers of these energy, power and transportation sectors need to identify which assets require attention, and what is the best action to take at what time. The choice becomes all the more complex as each asset type has different failure modes, and each failure may have different ways to influence the network. An ability to estimate the expected time to failure of assets will help the energy, power and transportation sectors make the optimal maintenance and replacement programs.

We will describe a Health Index model, which has been adopted by several energy, power and transportation sectors and enables their asset managers to oversee the health of all installed assets, recognizing the required additional maintenance or replacement need per asset, and representing the results as a function of time. [5, 6,7, 8$]$

The model combines Health Indexing and Risk Analysis, thereby providing full decision support on intervention actions to manage the power asset. The Health Index is a single indicator that represents the condition of an asset in relation to its specified performance and lifetime. The Health Index Tool is a dashboard for the asset manager and provides condensed information on the status of all relevant assets.

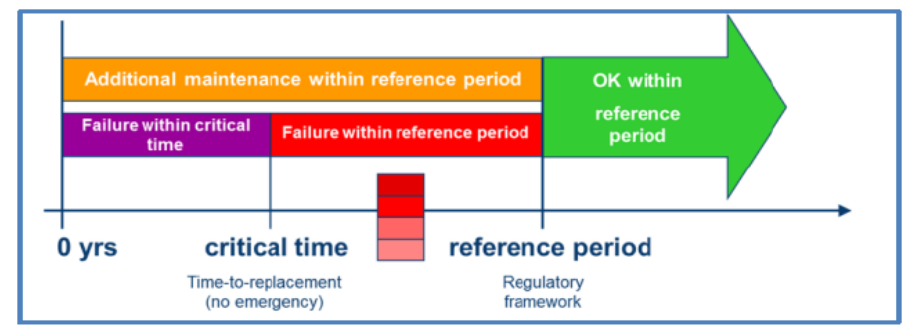

Fig. 5. Health Index Classification Scheme

The Health Indexing Methodology developed by DNV GL assesses the Health Index as accurately as the available data allow, and presents the results in a clear way, enabling the user to interpret the results in a blink of an eye. A simple colour scheme has been selected to represent asset health categories, each colour directly representing the required replacement or required additional maintenance need and urgency. A reference period is defined by the user, as an analysis horizon. Further to this, a critical time period is used to distinguish critical replacements from regular replacements. The reference period may be tuned to the regulator review period, or to any other relevant timeframe, and is typically 10 to 15 years. The critical time is set to the time required for a "standard" replacement plan, typically 1 to 3 years. These timeframes are defined as health classes as indicated in Figure 5. These classes are characterized by the colours green, orange, red and purple, which represent "good condition", "additional maintenance required", "replacement within reference period" and "immediate replacement" respectively. 
Health index assessment for a single component

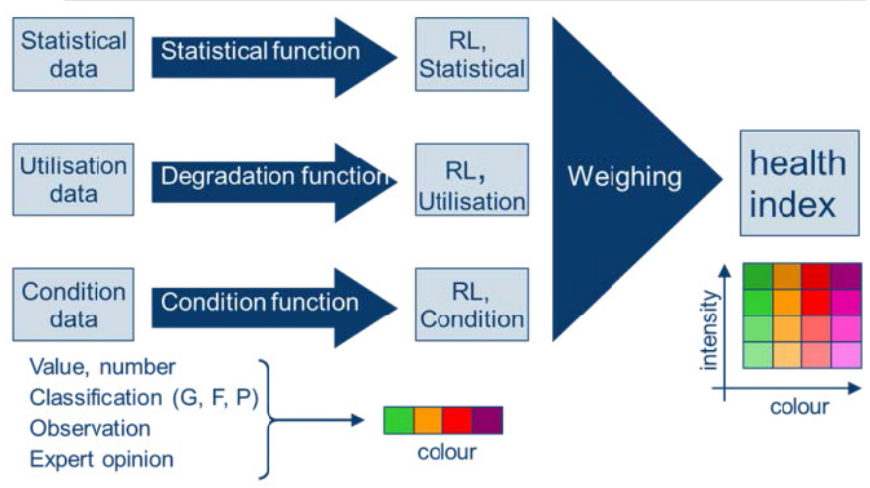

Fig. 6. Health Index Concept Using Three Types of Assessment Functions

The core of the Health Index Model is formed by the assessment functions that estimate the remaining life of the asset from available data. This estimation is based on whatever information is available, be it asset type, failure data, age, utilization data, maintenance and condition data. The most direct way of assessing the remaining life is direct condition data. However, often sufficient data are not available. Under such a situation, alternatives are built in, based on statistical information or operational (utilisation) information. In other words, if the remaining life cannot be assessed directly, the model produces an estimation based on statistical expectations, or on expected degradation due to stress.

As a result, three different types of assessment functions are used as shown graphically in Figure 6, i.e.:

- the statistical remaining life function;

- the degradation (utilisation) remaining life function; and

- the condition remaining life function.

Each of the above assessment functions estimates the most likely remaining life of the asset under study.

Energy, power and transportation sectors may also use a riskbased Asset Management System to support decision making in managing the power assets. To run a risk-based Asset Management model, there is a need to estimate the risks the assets are facing. Ideally, the risk of all assets shall be estimated individually, enabling a prioritization of all assets requiring attention.

In order to apply the Health Index model to risk-based decision support, the model is extended to include a Risk Index. The Health Index, estimating the remaining life of assets based on failure probability, is combined with the assets criticality or failure impact, thereby opening the option to plot all assets in a risk matrix, tuned to the specific power utility's core business values and circumstances.

To match best practice Asset Management processes, this risk matrix can be defined for the typical most relevant business values and KPIs such as reliability (frequency and duration of service disruptions), safety (number of accidents / injured persons) and financial (revenue lost/money spent). For example, the reliability impact of failure may be estimated by the load and number of customers connected to the individual assets and the system topography, like redundancy applications. The safety impact may be analysed using the probability of fire and explosion per asset type, in combination with the asset's housing, type of substation and distance to the public areas.

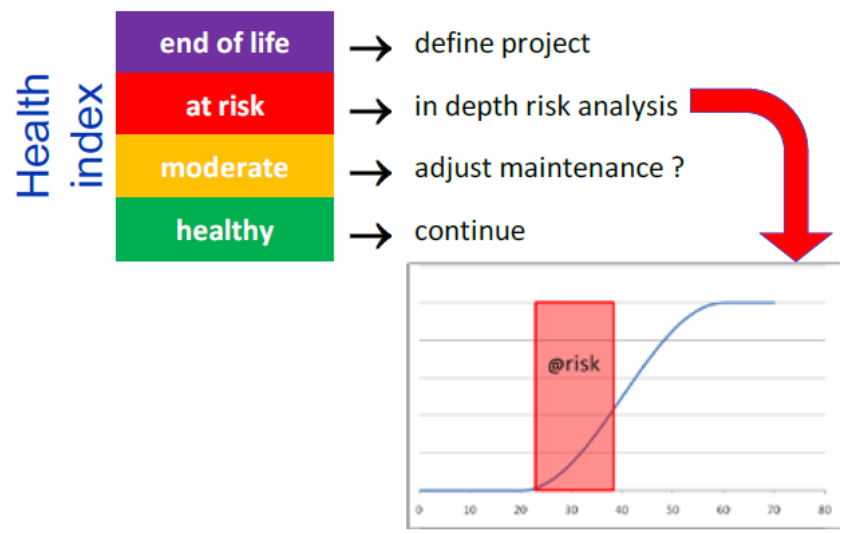

Fig. 7. Focused In-Depth Risk Analysis

With the risk matrix in place, and the health and criticality indices available, it is possible to plot all assets into the risk matrix and identify the assets that require urgent and critical attention. Assets that require attention may then be prioritized in terms of both on safety and reliability.

Once the assets with the highest risks are identified, individual in-depth analysis can be performed on selected assets, if required. For assets in the purple category, replacement is required as soon as possible. In case of a high level of uncertainty, the asset may require further detailed analysis before a replacement decision is made; whereas, assets with a low level of uncertainty may be replaced with minor or without risk analysis.

For assets in the red category, a longer timeframe is available for more detailed studies and condition assessment. This enables focused in depth analysis if needed to better substantiate a decision. Figure 7 gives a graphical representation of this principle.

The Health and Risk Indexing approach is part of the Asset Management decision cycle, an example of which is shown in Figure 8 .

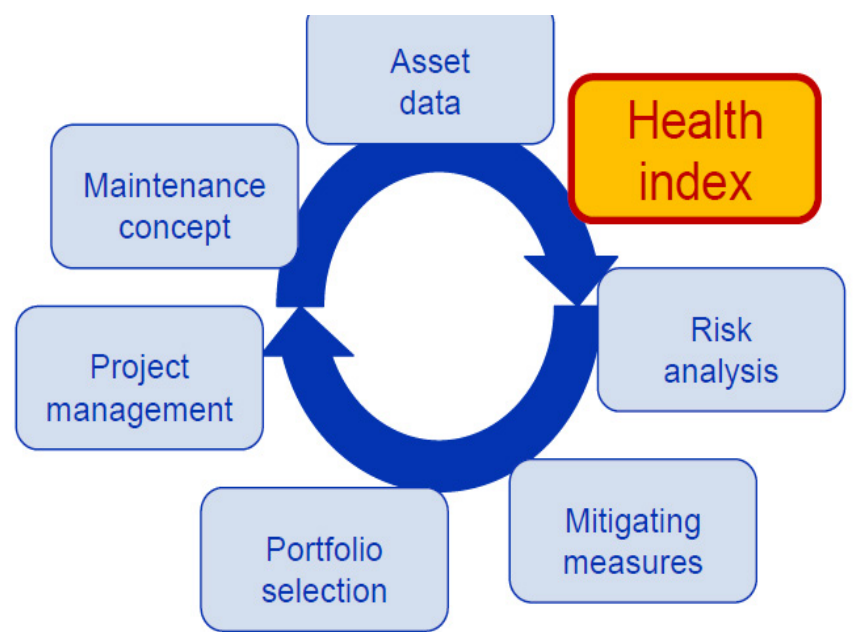

Fig. 8. Health Indexing as part of the Asset Management Cycle

Health and Risk Indexing processes data to produce information to decide what measures are required with what urgency and priority. The way in which it is embedded in the Asset Management process differs from user to user, but often it consists 
of a two-step approach. The first step, as indicated in Figure 7, divides the assets in one of four categories based on the remaining life and the additional activities required to survive the reference time period. The assets that score excellent or very poor need no detailed further analysis as they will be either continued, or replaced on short notice. For the other assets, before deciding on the basis of lifetime (equivalent to failure probability), the impact of failure is assessed, yielding a risk ranking.

Starting with the highest risk, solutions can then be generated. Each solution is again ranked on the basis of Solution Added Value. Finally, the asset manager will decide on a prioritization in the portfolio of solutions. The highest risk does not necessarily lead to the highest priority as this also depends on the cost and risk reduction potential of the solution.

After prioritization of the solutions, the asset manager will be able to select and plan the actions. For this he will use the ranking obtained. However, the decision will of course be influenced by the budget available and by the (un)acceptability of the risks assessed. Some actions are mandatory for meeting the power utility's directives and policies, and others may be highly relevant but not mandatory for the short term.

This will ultimately develop asset management strategy and policy leading towards reliability-centred maintenance for critical assets.

\section{Asset Management System Based on Reliability-Centered Maintenance}

Reliability-Centered Maintenance (RCM) is a structured process to determine the maintenance strategies. Various maintenance strategies have been evolved over time, i.e. Reactive maintenance (RM), Preventive maintenance (PM), Predictive maintenance (PdM). RM and PM approaches have been implemented in the earlier stage of maintenance strategies. However, these strategies were recognized as ineffective methodologies in 1960s because they do not capture the asset condition and the impacts and risks of the failures. Soon after, RCM has been first introduced by Nowlan in 1978 and applied to the Department of Defense of the US. Thanks to its effectiveness, RCM has been applied widely to other industries.

An example of RCM hierarchy is shown below:

Table 1: An example of RCM Approach

\begin{tabular}{|l|l|l|}
\hline \multicolumn{1}{|c|}{$\begin{array}{c}\text { Reactive } \\
\text { Element } \\
\text { Applications }\end{array}$} & \multicolumn{1}{|c|}{$\begin{array}{c}\text { Preventive } \\
\text { Element } \\
\text { Applications }\end{array}$} & $\begin{array}{c}\text { Predictive Element } \\
\text { Applications }\end{array}$ \\
\hline $\begin{array}{l}\text { Small parts and } \\
\text { equipment }\end{array}$ & $\begin{array}{l}\text { Equipment subject } \\
\text { to wear }\end{array}$ & $\begin{array}{l}\text { Equipment with } \\
\text { random failure } \\
\text { patterns }\end{array}$ \\
\hline $\begin{array}{l}\text { Non-critical } \\
\text { equipment }\end{array}$ & $\begin{array}{l}\text { Consumable } \\
\text { equipment }\end{array}$ & Critical equipment \\
\hline $\begin{array}{l}\text { Equipment } \\
\text { unlikely to fail }\end{array}$ & $\begin{array}{l}\text { Equipment with } \\
\text { known failure } \\
\text { patterns }\end{array}$ & $\begin{array}{l}\text { Equipment not } \\
\text { subject to wear }\end{array}$ \\
\hline $\begin{array}{l}\text { Redundant } \\
\text { systems }\end{array}$ & $\begin{array}{l}\text { Manufacture } \\
\text { recommendations }\end{array}$ & $\begin{array}{l}\text { Systems which failure } \\
\text { may be included by } \\
\text { incorrect preventive } \\
\text { maintenance }\end{array}$ \\
\hline
\end{tabular}

In terms of methodologies, the three maintenance strategies, i.e. RM, PM and PdM, are broken down into four maintenance methodologies, i.e. Run-to-Fail maintenance, Time-based maintenance, Condition-based maintenance, and Risk-based www.astesj.com maintenance. Figure 9 below shows the relationship between the maintenance strategies and methodologies.

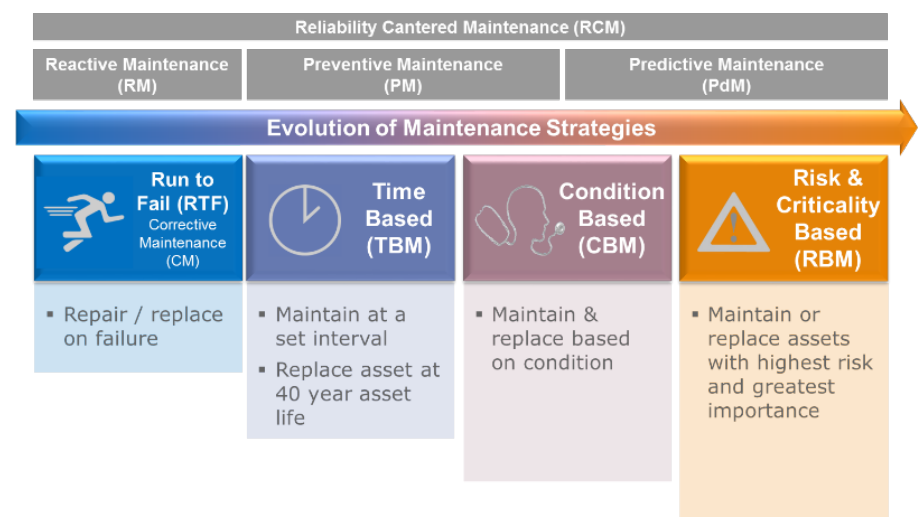

Fig. 9. Consistent and Technically Sound Process towards a Reliability-Centred Asset Management System

\section{The condition-based approach:}

The condition based approach bases on the understanding of asset's condition i.e. health index of the asset, which indicate how good or bad the condition of asset is. It also indicates the remaining life time of the asset, the likelihood of the asset's failure. The health index system enables asset managers to oversee the health of all installed assets, recognize the required additional maintenance or replacements per location and plotted in time. An example of asset health index result is shown in Figure 10 below.

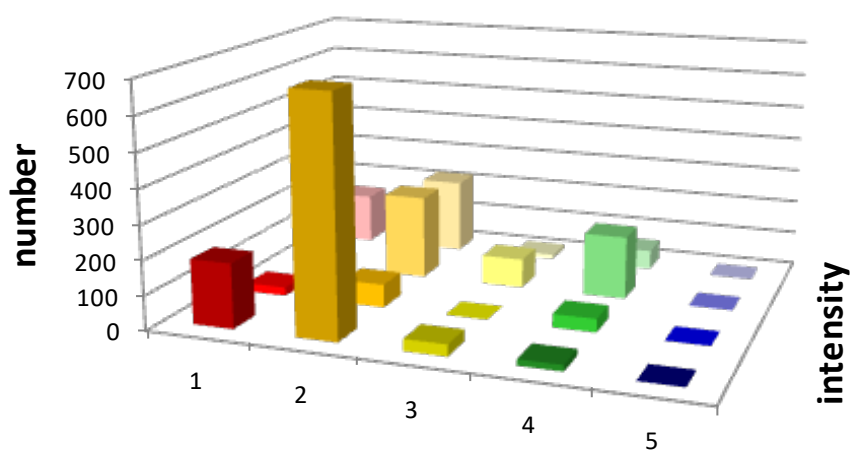

Fig. 10. Example of health index results of a fleet of assets

A simple color scheme presents the results indicating the required replacement or required additional maintenance attention. A reference period is agreed with the asset managers and usually a critical planning time period is defined to distinguish critical replacements from regular replacements. Based on these timeframes we can now define health classes as indicated in Error! Reference source not found.. The classes are characterized by the colors blue, green, yellow, orange, and red.

\section{Risk-based approach:}

Once the Health Index has been developed and determined for an asset, that asset can also be graded according to its Criticality of failing. Its Criticality can be determined with reference to all relevant asset management business values, like safety, reliability etc. The level of Criticality will be determined by the asset type, location, local grid situation etc. Once the Criticality and the Health Index are ready, an asset management risk matrix can be developed with on one axis the Criticality and on the other axis the Health Index. The Health Index gives an indication of remaining 
life, but it can also be seen as an indicator of probability of failure. Criticality indicates the impact of the failure. Combining both will show the risk level of each asset in the risk matrix. An example of a risk matrix is shown in Figure 11.

\begin{tabular}{|l|l|l|l|l|l|}
$\begin{array}{l}\text { Remaining } \\
\text { Life }\end{array}$ & Catastrophic & Severe & Moderate & Small & Negligible \\
\hline s1 year & Unacceptable & Unacceptable & High & High & Medium \\
\hline 2-3 years & Unacceptable & High & High & Medium & Low \\
\hline 3-7 years & High & High & Medium & Low & Low \\
\hline 7-15 years & High & Medium & Low & Low & Negligible \\
\hline$>15$ years & Medium & Low & Low & Negligible & Negligible \\
\hline
\end{tabular}

Fig. 11. Example of a risk matrix specifically for the health index

As a general observation, the following trend has started to emerge [9, 10, 11, 12]:

- $\quad$ Moving from time-based to condition-based maintenance and further from condition-based to risk-based maintenance for most critical assets - Using Fukushima Nuclear Power Station as an example, an incident in a nuclear power station can have dire consequences to the environment, human life, properties and the livelihood of the population in vicinity. The reliability-centred design and maintenance based on multiple KPIs will be the most appropriate choice in maintaining such an asset;

- Moving from risk-based Asset Management to Reliability-Centred Asset-Management, whereby

- Near-real-time information is captured and analysed; and

- Real-time failure prediction function plays a key role in decision making support.

- $\quad$ Moving from Big Data to Smart Data

- With a more comprehensive data considered in the Smarter-Asset-Management, the Big Data will be the next challenge in Asset Management;

- Smart Data is the next level of dealing with Big Data in a smarter and more effective way; and

- More advanced data analytics techniques will be adopted such as machine learning/artificial intelligence approach.

- $\quad$ Integrated Energy Asset Management System merging Asset Management System (AMS) with Energy Management System (EMS): - The merger between the EMS and AMS will help address the Big Data more effectively.

Energy, power and transportation sectors are moving in the data analytics era. This is the result of several global forces - one being the proliferation of less expensive electronic monitoring technologies and the speed and availability of communications systems. Furthermore, more and more energy, power and transportation sectors want to embark on developing the 'Smartest' asset systems possible. As a result, an unprecedented amount of raw data is being collected by energy, power and transportation sectors each day.

On the other hand, criticality and health of each of the assets are influenced by the asset operational environment. A systematic

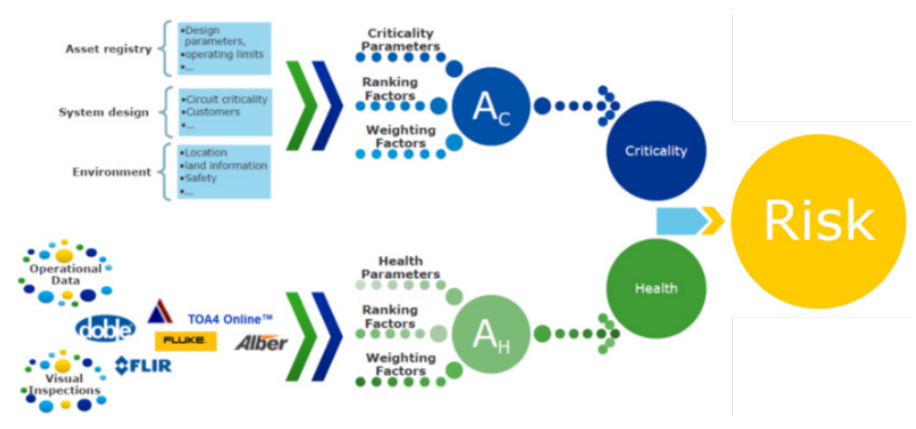

Fig. 12. Consistent and Technically Sound Process towards a Reliability-Centred Asset Management System

approach to assess the criticality and health will deliver a practical approach to risk assessment across the entire asset base.

Using the ranking factors and weighting factors, we are able to tailor the assessment to specific business priorities and objectives in achieving reliability-centred Asset Management strategy and policies.

As criticality and health are two essential factors in assessing asset risks, the trend mentioned in the start of this section enables the integration of Asset Management Systems with other IT systems, such as Geospatial Systems, distribution management systems, energy management systems, signalling and control systems, etc. will help these systems in:

- improving the accuracy in identifying critical contingencies by making use of more accurate component's criticality and utilization information;

- $\quad$ improving the performance by filtering out low failure probability;

- $\quad$ better big-data management by using a single database;

- improving the effectiveness by avoiding redundancy of assessments and processes; and

- $\quad$ optimising decision making processes based on a more comprehensive assessment considering both global and local aspects

\section{Conclusion}

The energy, power and transportation sectors need to move from time-based to Predictive Maintenance / Condition Based Maintenance of their asset and ultimately towards ReliabilityCentred Asset Management strategy and policies. This will involve:

- Apply Reliability-Centred Maintenance philosophy in selection of effective maintenance tasks;

- Apply Reliability-Centred Maintenance philosophy to determine what operational and conditional data is required;

- $\quad$ Reliability-centred maintenance provides a platform for developing an effective Reliability-Centred Asset Management strategy; and

- Lastly, a platform with appropriate software applications to ensure a sustainable Reliability-Centred Asset Management strategy. 
To achieve this state of managing the assets, particularly the critical asset, cost-effectively with minimum risks, energy, power and transportation sectors need to embark on a journey to develop resources and capability within the organisation to manage such changes. External helps from experts with in-depth knowledge, vast experience and up-to-date competency will definitely be necessary to leverage on the evolving trends in the ICT infrastructures.

\section{References}

[1] Institute of Asset Management: Asset Management - an anatomy (December 2011 version 1)

[2] "ISO 9001:2015 Quality management systems" standard

[3] "ISO 55001:2014 Asset Management" standard. BSI Group.

[4] Sungin Cho, Khanh-Loc Nguyen and Jos Wetzer, "Conceptual Design for Asset Management System under the Framework of ISO 55000", proceedings CIRED 23rd International Conference on Electricity Distribution Lyon, 15-18 June 2015, Paper 1025

[5] Mischa Vermeer, Ashutosh Sharma, Jos Wetzer, Peter Zonneveld and Dirk Boender, "Asset Management Decision Support Modelling, using a Health Index, for Maintenance and Replacement Planning", proceedings The 9th GCC - CIGRE International Conference, 2013

[6] Mischa Vermeer and Jos Wetzer, "Transformer health and risk indexing, including data quality management", Proceedings 45th CIGRÉ Session, 2014, Paper A2-116

[7] Fred Steennis and et al, "The Intriguing Behavior over Time of PD's from Defects in MV Cables and Accessories; Lessons Learned with SCG, an OnLine Monitoring System", proceedings CIRED $21^{\text {st }}$ International Conference on Electricity Distribution, 2011

[8] Mischa Vermeer and et al, "Asset Management Decision Support Modeling, using a Health Index, for Maintenance and Replacement Planning", PowerTech, 2015 IEEE Eindhoven, 2015

[9] Khanh-Loc Nguyen, Liang Hongzhu Daniel, Kelvin Tan, "Energy Asset Management System (EAMS) - Preparation for future power system with high penetration of DGs and RE", ACEF Manila Philippines, 2015 June. Presentation online: http://d335hnnegk3szv.cloudfront.net/wpcontent/uploads/sites/837/2015/06/KEMCO-DDWPresentation_Session-IIKhanh-Loc-Nguyen.pdf

[10] Khanh-Loc Nguyen, Liang Hongzhu Daniel, Kelvin Tan, "Energy Asset Management System - A Hybrid Solution for Future Distribution System with High Penetration of DGs and RE", WES (World Engineers Summit), Track 3, 2015 July, Singapore. Online link: http://www.wesies.org/summit/tracks/

[11] Khanh-Loc Nguyen, Liang Hongzhu Daniel, Kelvin Tan, "Energy Asset Management System for offshore rigs", SIEW ACES Singapore, 2015 October

[12] Khanh-Loc Nguyen, Daniel Liang, Terence Goh, Kelvin Tan, “A smart asset management method for offshore rigs", EASEC-14 Conference, Vietnam, 2016 January. Online link: http://easec14.hcmut.edu.vn/wpcontent/uploads/2015/06/S4-Summary-and-list-of-papers.pdf 